\title{
Estereotipos sobre el envejecimiento, temores y percepción sobre la felicidad: un análisis sociodemográfico
}

\author{
Stereotypes about aging, fears and perception about happiness: a \\ sociodemographic analysis
}

\author{
Verónica López Fernández ${ }^{1}$ \\ Universidad Internacional de La Rioja (España) \\ Solana Salessi ${ }^{2}$ \\ Universidad Nacional de Rafaela (Argentina) \\ Karen Johana González Restrepo ${ }^{3}$ \\ Secretaría de Educación del Distrito Capital Bogotá (Colombia)
}

Recibido: $29-04-20$

Aceptado: $12-06-20$

\section{Resumen}

La presente investigación se orientó a explorar las vinculaciones entre los estereotipos negativos sobre el envejecimiento, los temores respecto a la vejez, la satisfacción con la vida y ciertas características sociodemográficas. Con tal propósito, se diseñó un estudio empírico cuantitativo transversal, de alcance descriptivo-correlacional. Se conformó una muestra de 314 españoles ( $74 \%$ mujeres; $M_{\text {edad }}=38$ años), seleccionados según un muestreo por disponibilidad. Los participantes respondieron una selección de instrumentos para medir los constructos bajo estudio. Sobre los datos reunidos se ejecutaron análisis descriptivos y correlacionales. Los resultados muestran que no hay diferencias en ninguna de las variables estudiadas en función del sexo, pero sí se observan mayores estereotipos negativos entre quienes conviven con adultos mayores. La edad se asocia negativamente tanto a los estereotipos y temores como a la satisfacción con la vida. Finalmente, los estereotipos negativos sobre el envejecimiento se vinculan con menores niveles de satisfacción vital proyectada a cinco años. Se discuten los hallazgos y señalan direcciones para futuros estudios en el área.

Palabras clave: Envejecimiento; estereotipos; temores; satisfacción con la vida.

\footnotetext{
${ }^{1}$ Doctora en Psicología y Ciencias de la Educación. Coordinadora del Departamento de Psicología de la Educación y Psicobiología. Autor para correspondencia. E-mail: veronica.lopez@unir.net ORCID: https://orcid.org/0000-0003-0483-5884

${ }^{2}$ Doctora en Psicología. Docente en la Universidad Nacional de Rafaela. E-mail: solanasalessi@unraf.edu.ar ORCID: https://orcid.org/0000-0001-9496-9493

${ }^{3}$ Máster en Neuropsicología y Educación. Docente en la Secretaría de Educación del Distrito Capital Bogotá. E-mail: kgonzalez1@educacionbogota.edu.co. ORCID: https://orcid.org/0000-0002-6711-6249
}

(C) Los autores. Este artículo es publicado por la Revista de Investigación en Psicología de la Facultad de Psicología, Universidad Nacional Mayor de San Marcos. Este es un artículo de acceso abierto, distribuido bajo los términos de la licencia Creative Commons Atribucion - No Comercia_Compartir Igual 4.0 Internacional. (http://creativecommons.org/licenses/by-nc-sa/4.0/) que permite el uso no comercial, distribución y reproducción en cualquier medio, siempre que la obra original sea debidamente citada. 


\begin{abstract}
The present investigation was oriented to explore the links between negative stereotypes about aging, fears about old age, satisfaction with life and certain sociodemographic characteristics. For this purpose, a cross-sectional quantitative empirical study of descriptive-correlational scope was designed. A sample of 314 Spaniards was formed ( $74 \%$ women, $\mathrm{M}_{\text {edad }}=38$ years), selected according to a sampling by availability. The participants answered a selection of instruments to measure the constructs under study. On the collected data, descriptive and correlational analyzes were carried out. The results show that there are no differences in any of the variables studied according to sex, but there are greater negative stereotypes among those who live with older adults. Age is negatively associated with both stereotypes and fears and satisfaction with life. Finally, negative stereotypes about aging are linked to lower levels of life satisfaction projected to five years. The findings are discussed and directions for future studies in the area are indicated.
\end{abstract}

Keywords: Aging; stereotypes; fears; satisfaction with life.

El envejecimiento se refiere a la etapa final del proceso evolutivo, donde se produce una merma significativa del nivel óptimo de funcionamiento orgánico y mental de una persona (González \& de la Fuente, 2014). Se trata de una realidad innata del ser humano de difícil aceptación para muchas personas; tal vez, precisamente, por la disminución de determinadas capacidades funcionales. No obstante, el envejecimiento es un proceso continuo de carácter multidimensional y multidireccional, puesto que obedece a numerosos factores externos e internos al individuo (Alvarado \& Salazar, 2014). Constituye un proceso individual que no se equipara exclusivamente ni con la edad cronológica, ni con la edad biológica ni con la edad social; sino que, por el contrario, cada una de estas "edades" converge con la subjetividad de la persona, imprimiendo un sello particular en cada sujeto (González \& de la Fuente, 2014).

En virtud de su complejidad, el envejecimiento debe ser concebido desde diversos enfoques: biológico, demográfico, psicológico, familiar y sociocultural (Diaz, et al., 2015). En efecto, en la vejez juegan un papel fundamental el contexto psicosocial, los recursos económicos, los aspectos sociohistóricos y las variables culturales (López \& Marín, 2016). En este sentido, cada sociedad sustenta una concepción diferente sobre el envejecimiento. Las representaciones e impresiones sociales suscitan imaginarios y estereotipos que, en las sociedades occidentales son generalmente negativos, dando lugar a sentimientos de aversión hacía la vejez como etapa natural del ciclo vital (Alvarado \& Salazar, 2014).

Los estereotipos constituyen percepciones o creencias falsas, que se perciben como ciertas. Suponen generalizaciones no científicas de carácter subjetivo, capaces de influir sobre las conductas, emociones y actitudes de las personas (Perea, 2018). En este sentido, mientras que las impresiones positivas sobre el envejecimiento permiten acercarse a esta etapa de forma exitosa, manteniendo la 
actividad social, la autoestima, la calidad de vida y el adecuado funcionamiento biopsicosocial; los estereotipos negativos pueden reducir la satisfacción y modificar la percepción de las propias capacidades funcionales (Kleinspehn-Ammerlahn, Kotter-Grühn, \& Smith, 2008). Así, por ejemplo, Pearman, Hertzog y Gerstorf (2014) encontraron que las quejas de memoria de las personas mayores no están relacionadas con la edad, sino con los estereotipos sobre la vejez.

Los contextos en los cuales se desenvuelve el adulto mayor tienen un papel crucial en el proceso de envejecimiento, desde el momento que pueden influir sobre las autopercepciones, otorgándole un margen objetivo sobre su propio proceso evolutivo (Kleinspehn-Ammerlahn, et al., 2008). En este sentido, las percepciones que los adultos mayores tienen sobre este proceso tienden a ser influidas por lo que la sociedad opina. Tal es así que frente a atribuciones negativas pueden sucederse desplegarse conductas de segregación y marginación. El estereotipo subraya la decadencia a nivel físico y mental, lo que finalmente produce un rechazo sobre este proceso natural del ciclo vital (Jensen \& Vogel, 2005). En efecto, se ha señalado (Dionigi, 2015) que los estereotipos de envejecimiento que se relacionan a la declinación de las funciones tienden a reforzar el potencial del envejecimiento, siendo, así, un proceso de difícil aceptación frente a los temores que motiva.

El estudio de las vinculaciones entre las características sociodemográficas y los estereotipos sobre el envejecimiento presenta resultados contrapuestos. En lo que respecta al sexo, por ejemplo, León y Montero (2015) no encontraron diferencias; en tanto que Allan y Johnson (2009) informaron que las mujeres presentaban menos estereotipos negativos que sus contrapartes varones. En lo que hace a la edad, Lasagni Colombo et al. (2013) han informado que los jóvenes universitarios muestran más estereotipos que las personas adultas; aunque éstos también están presente en estas edades. En línea con tales evidencias, ArnoldCathalifaud, Thumala, Urquiza y Ojeda (2007) han encontrado que los jóvenes tienen percepciones más negativas que subrayan la condición de vulnerabilidad del adulto mayor, y retroalimentan estereotipos referentes a la disminución de la funcionalidad orgánica y las dificultades de adaptación. No obstante, entre los niños y preadolescentes lejos de primar las connotaciones ligadas a la enfermedad o la incapacidad, el estereotipo de vejez se centra en los aspectos psicológicos del adulto mayor o su espacio dentro de un grupo social (Monchietti, Lombardo \& Sánchez, 2015). En este sentido, Oscanoa, Romero-Ortuno y Cruz-Jentoft (2016) afirman que estar expuestos a estereotipos negativos sobre el envejecimiento produce efectos negativos en el sistema cardiovascular, y produce lo que se denomina el efecto de coincidencia, que significa que exponerse a dichos estereotipos de índole negativa relacionados con la función física o cognitiva, coincide a posteriori con los resultados en la evaluación de ambas dimensiones. 
En lo que hace a las relaciones entre los estereotipos negativos y otras variables sociodemográficas, Lasagni Colombo et al. (2013) informaron vinculaciones significativas con el nivel educativo y la ocupación, pero no con el estado civil de los participantes o con el hecho de convivir con personas mayores. Concretamente, los autores informaron de que los trabajadores remunerados y los jubilados presentan menos estereotipos negativos que aquellas personas que no trabajan y las que trabajan de forma no renumerada. En cuanto al nivel educativo, se encontró que a mayor escolaridad había menor incidencia de estereotipos negativos (Lasagni Colombo et al., 2013). En contraposición, Rello, Bravo y Plata (2018) encontraron diferencias significativas en los estereotipos dependiendo de si las personas convivían o no con adultos mayores, subrayando el rol de la calidad del tipo de contacto, antes que la cantidad de los mismos.

En relación a los estereotipos negativos, cabe reseñar que las creencias falsas y mitos derivan en muchas ocasiones en temores hacia el propio proceso de envejecimiento, y dichos temores generan negación y hostilidad (Amico, 2009). Por lo tanto, si una persona hace suya la creencia falsa de un estereotipo, asumirá que con el paso de los años el declive es inevitable, modificando su conducta e incluso dejando de hacer actividades y de participar en la sociedad, generando insatisfacción con la vida. Desde teorías de la psicología cognitiva como la de Fiske y Neuberg, los estereotipos, al aglutinar información sobre las características de un elemento concreto ponen el énfasis en la importancia de tomar en consideración los propios procesos cognitivos implicados (Bart-Tal., 1994). Esto implica que los estereotipos fruto de la categorización de las características del envejecimiento pueden tener un gran efecto en el ámbito conductual y emocional.

Estudios como el de Pinazo-Hernandiz, Torregrosa-Ruiz, Jimenez-Marti y Blanco-Molina (2019) demuestran la importancia de la participación de las personas mayores en actividades y de la relación que existe entre esta y la satisfacción con la vida, por lo que es fundamental abordar el concepto de temores, relacionado con los estereotipos y con la satisfacción.

Los antecedentes relevados muestran que las evidencias en materia de vinculación entre estereotipos y variables sociodemográficas distan de ser concluyentes, por lo que es fundamental que nuevos estudios retomen y profundicen esta línea de investigación teniendo en cuenta como se ha comentado que el envejecimiento es un proceso continuo de carácter multidimensional y multidireccional, que obedece a numerosos factores externos e internos al individuo (Alvarado \& Salazar, 2014), y que constituye un proceso individual que no se equipara exclusivamente ni con la edad cronológica, ni con la edad biológica ni con la edad social; sino que se relaciona más bien con un sello particular en cada sujeto (González \& de la Fuente, 2014).

. Frente a este panorama, el presente estudio se orientó a analizar la relación entre los temores hacia el envejecimiento, los estereotipos de vejez, la satisfacción 
vital percibida y algunas variables demográficas, siendo los interrogantes que movilizan esta investigación los siguientes: ¿cuáles son las diferencias en estereotipos y temores entre hombres y mujeres?; ¿cuáles son las relaciones entre la edad, los estereotipos negativos sobre el envejecimiento y los temores hacia la vejez?; ¿cuál es la relación entre la satisfacción con la vida y los estereotipos y temores respecto de la vejez y el envejecimiento?; ¿existen diferencias significativas en los estereotipos y temores en función del tamaño de la localidad de residencia?; ¿cuál es la relación entre el nivel de estudios, los estereotipos, los temores y la satisfacción vital; ¿cuáles son las diferencias en los temores, estereotipos y nivel de satisfacción entre quienes conviven y no conviven con un adulto mayor?.

Conocer las respuestas a estas preguntas nos permitirá avanzar más en el logro de un envejecimiento más individualizado y activo. Por todo ello, los objetivos del presente trabajo son: conocer los estereotipos negativos hacia la vejez, los temores relacionados con el envejecimiento y la satisfacción con la vida en una muestra de personas de diferentes edades, formación, y otras variables sociodemográficas, con el fin de analizar cómo se comportan dichas variables y se relacionan entre sí.

\section{MÉTODO}

\section{Diseño}

Para dar respuesta a las cuestiones comentadas, la presente investigación corresponde a un estudio empírico, cuantitativo y transversal. Se enmarca en los lineamientos de la estrategia descriptivo-asociativa (Ato, López, \& Benavente, 2013), ya que su finalidad fue explorar la relación entre un selecto grupo de variables, así como potenciales diferencias a la luz de ciertas las características sociodemográficas de los participantes.

\section{Participantes}

Se apeló a un muestreo no probabilístico, por disponibilidad. Participaron del estudio 314 jóvenes y adultos residentes en diversas ciudades del norte, centro, sur y este de España. El 52\% de la muestra residía en localidades con más de 100.000 habitantes. La edad promedio fue de 38 años (DT=11.51). El $61 \%$ de los participantes tenían estudios superiores universitarios. El 74\% de los participantes eran mujeres. Sólo el $18 \%$ convivía con personas adultas mayores de 65 años, en tanto que el $82 \%$ se relacionaba cotidianamente con ellas.

\section{Procedimiento}

La recolección de los datos se llevó a cabo durante el segundo semestre del año 2018. Todos los participantes fueron contactados a través de un formulario electrónico. Luego de informales sobre los objetivos del estudio se los invitó a 
responder, previa firma de un protocolo de consentimiento informado. La participación fue voluntaria, anónima y confidencial. No se brindaron incentivos de ningún tipo.

\section{Instrumentos}

Estereotipos negativos hacia la vejez. Se evaluaron mediante el Cuestionario de Estereotipos Negativos hacia la Vejez (CENVE) desarrollado por Mena, Sánchez Palacios y Trianes (2005). El instrumento se compone por 15 ítems, presentados en formato tipo Likert de 4 puntos ( $1=$ Muy en desacuerdo, $2=$ Algo en desacuerdo, $3=$ Algo de acuerdo, 4= Muy de acuerdo). La puntuación oscila entre 5 y 20 puntos para cada una de las tres subescalas que integran el cuestionario: (a) estereotipos de salud (5 ítems, ej: "la mayor parte de las personas, cuando llegan a los 65 años de edad, aproximadamente, comienzan a tener un considerable deterioro de memoria"; $\alpha=.75$ ); (b) estereotipos motivacionales-sociales (5 ítems, ej: "la mayor parte de las personas, cuando llegan a los 65 años de edad, aproximadamente, pierden interés por las cosas"; $\alpha=$.74) y; (c) estereotipos de carácter-personalidad (5 ítems, ej: "la mayor parte de las personas, cuando llegan a los 65 años de edad, aproximadamente, se vuelven más rígidas e inflexibles"; $\alpha=.71$ ). Puntuaciones altas indican un elevado grado de creencia en los estereotipos negativos de la vejez. Según los autores, el instrumento obtuvo una consistencia interna buena $(\alpha=0,89)$ en el estudio de validación y en otras investigaciones también ha mostrado un nivel de consistencia interna elevado $(\alpha=0,85)$, indicando una buena fiabilidad del instrumento (Brotons Rodes, Lorente Martínez y Sitges Maciá, 2020). Evidencias de validez de estructura interna obtenidas en nuestro presente estudio muestran un adecuado ajuste a un modelo trifactorial oblicuo $\left(\mathrm{SB} \chi^{2}=1.59, \mathrm{GFI}=.90, \mathrm{CFI}=.91\right.$, RMSEA $=0.04[0.03 ; 0.05])$.

Temores hacia el envejecimiento. Se exploraron mediante cuatro ítems (Fundación Humanismo y Ciencia, 2017); a saber: "temo que cuando sea mayor, todos mis amigos se habrán ido"; "la idea de sobrevivir a mi pareja me asusta"; "siempre temía el día en que me mirara al espejo y encontrara una cana"; y "cuanto mayor me hago, más ansioso me vuelvo sobre mi futuro". Los mismos fueron presentados en formato tipo Likert de 5 puntos $(1=$ Totalmente en desacuerdo, $2=$ En desacuerdo, $3=\mathrm{Ni}$ de acuerdo ni en desacuerdo, $4=$ De acuerdo, $5=$ Totalmente de acuerdo) y mostraron adecuado nivel de consistencia interna $(\alpha=.71)$. Se obtuvo un puntaje general, a sabiendas que puntuaciones altas indican un elevado temor hacia el envejecimiento. Evidencias de validez de estructura interna obtenidas en el presente estudio muestran un adecuado ajuste a un modelo unifactorial ( $\mathrm{SB} \chi 2=$ $2.41, \mathrm{GFI}=.90, \mathrm{CFI}=.90, \mathrm{RMSEA}=0.03[0.02 ; 0.04])$.

Satisfacción con la vida. Se examinó mediante la técnica "escalera de la vida" (Rojas, 2008). De acuerdo a esta técnica el sujeto evaluado debe imaginar escalones numerados de 0 a 10. Con base en esta escala responder: (a) ¿en qué 
lugar de la escalera sientes que estás en este momento tu vida? Y; (b) ¿en qué escalón crees que estarás dentro de 5 años? A mayor puntuación en ambas preguntas, más satisfacción vital presente y proyectada.

Variables sociodemográficas. Se recabó información sobre la edad, el sexo, el nivel de formación, la localidad de residencia, la cantidad de habitantes, la convivencia y relación cotidiana con una persona mayor de 65 años.

\section{Consideraciones éticas}

El estudio cumplió los estándares éticos requeridos en la investigación con seres humanos, tal como lo establece la Asociación Americana de Psicología (2017).

\section{Estrategia de análisis de los datos}

Inicialmente, se realizó un análisis de datos perdidos valorando su proporción y la posible presencia de sesgos en su distribución. Para ello, se aplicó el test conjunto de aleatoriedad de Little. Se calcularon los índices descriptivos (medias y desvíos típicos). Para responder los interrogantes de investigación Se realizaron pruebas de diferencias de medias ( $t$ de Student); análisis de varianza (ANOVA de un factor) y; análisis de correlaciones bivariadas, según corresponda. Para el análisis de los datos se utilizó el paquete estadístico SPSS (versión 21).

\section{RESULTADOS}

El porcentaje de datos perdidos en cada ítem no superó el 5\%. La prueba de Little indicó que el patrón de valores perdidos respondía completamente al azar (MCAR; $\left.\chi_{(144)}^{2}=152.08, p=.215\right)$. Estos datos fueron reemplazados matemáticamente por valores calculados a partir del método EM (expectación-maximización). La Tabla 1 presenta los estadísticos descriptivos y pruebas $t$ de Student efectuadas con el fin de analizar posibles diferencias entre varones y mujeres

\section{Tabla 1}

Estadísticos descriptivos y pruebas $t$ de Student para género y convivencia $(n=314)$

\begin{tabular}{lcccccccccc}
\hline & \multicolumn{2}{c}{ Varón } & \multicolumn{2}{c}{ Mujer } & \multicolumn{3}{c}{ No convive } & \multicolumn{2}{c}{ Convive } \\
& $\mathbf{X}$ & $\mathbf{D T}$ & $\mathbf{X}$ & $\mathbf{D T}$ & $\mathbf{t}_{(314 ; 312)}$ & $\mathbf{X}$ & $\mathbf{D T}$ & $\mathbf{X}$ & $\mathbf{D T}$ & $\mathbf{t}_{(314 ; 312)}$ \\
\hline 1. Estereotipos sobre salud & 9.84 & 2.33 & 9.85 & 3.63 & $.86 \mathrm{~ns}$ & 9.47 & 2.53 & 10.31 & 2.58 & $-2.22^{*}$ \\
2. Estereotipos sobre motivación & 9.55 & 2.29 & 9.40 & 2.56 & $.45 \mathrm{~ns}$ & 9.27 & 2.47 & 10.22 & 2.45 & $-2.61^{* *}$ \\
3. Estereotipos sobre personalidad & 10.49 & 2.40 & 10.07 & 2.90 & $1.14 \mathrm{~ns}$ & 10.10 & 2.79 & 10.52 & 2.72 & $-1.00 \mathrm{~ns}$ \\
4. Temores hacia la vejez & 7.0 & 2.22 & 7.37 & 2.47 & $-1.05 \mathrm{~ns}$ & 7.24 & 2.42 & 7.48 & 2.35 & $-.67 \mathrm{~ns}$ \\
5. Felicidad actual & 6.33 & 2.25 & 7.11 & 1.73 & $-1.58 \mathrm{~ns}$ & 7.07 & 1.81 & 6.75 & 1.82 & $.21 \mathrm{~ns}$ \\
6. Felicidad futura & 7.42 & 2.26 & 7.81 & 1.69 & $-1.69 \mathrm{~ns}$ & 7.86 & 1.72 & 7.09 & 1.96 & $2.96^{* *}$ \\
\hline
\end{tabular}

Nota: $\mathrm{ns}=$ no significativo; $* \mathrm{p}<.05 ; * * \mathrm{p}<.01$ 
De la tabla precedente se desprende que no se encontraron diferencias estadísticamente significativas según el sexo ni en los estereotipos negativos, ni en los temores hacia el envejecimiento, ni en ninguna de las preguntas relativas a la felicidad o satisfacción vital. Sin embargo, sí se encontraron en los estereotipos referidos a la salud y a la motivación entre los participantes que conviven y no conviven con mayores de 65 años; siendo superiores en los primeros.

La Tabla 2 informa las correlaciones entre las variables estudiadas. Como puede observarse, se la edad correlacionó negativamente con los estereotipos negativos referidos a la personalidad del adulto mayor, y con los temores hacia el envejecimiento; así como con la satisfacción con la vida. A su vez, las tres formas de estereotipos mostraron asociaciones negativas con la satisfacción vital proyectada a cinco años.

\section{Tabla 2}

Coeficientes $r$ de Pearson entre las variables estudiadas $(\mathrm{n}=314)$

\begin{tabular}{|c|c|c|c|c|c|c|c|}
\hline Variables & 1 & 2 & 3 & 4 & 5 & 6 & 7 \\
\hline 1. Estereotipos sobre salud & - & $.69 * *$ & $.74^{* *}$ & $.46^{* *}$ & $-.06 \mathrm{~ns}$ & $-.12 *$ & $-.07 \mathrm{~ns}$ \\
\hline 2. Estereotipos sobre motivación social & & - & $.76^{* *}$ & $.45^{* *}$ & $-.04 \mathrm{~ns}$ & $-.16^{* *}$ & $-.02 \mathrm{~ns}$ \\
\hline 3. Estereotipos sobre personalidad & & & - & $.50 * *$ & $-.04 \mathrm{~ns}$ & $-.12 *$ & $-.16^{* *}$ \\
\hline 4. Temores hacia el envejecimiento & & & & - & $-.12 *$ & $-.09 \mathrm{~ns}$ & $-.22 * *$ \\
\hline 5. Felicidad actual & & & & & - & $.73^{* *}$ & $-.12 *$ \\
\hline 6. Felicidad futura & & & & & & - & $-.35 * *$ \\
\hline 7. Edad & & & & & & & - \\
\hline
\end{tabular}

Nota: $\mathrm{ns}=$ no significativo; $* \mathrm{p}<.05 ; * * \mathrm{p}<.01$

Finalmente, los análisis ANOVA no arrojaron diferencias significativas en función de la cantidad de habitantes por localidad (Estereotipos sobre salud: $\mathrm{F}_{65}$ 309)= 2.08, $p=.076$; Estereotipos sobre motivación: $\mathrm{F}_{(5 ; 309)=} 1.29, p=.264$; Estereotipos sobre personalidad: $\mathrm{F}_{(5 ; 309)=} 1.40, p=.221$; Temores hacia la vejez: $\mathrm{F}_{(5 ; 309)=} 1.16$, $p=.324$; Satisfacción con la vida actual: $\mathrm{F}_{(5 ; 309)=1} 1.119, p=.312$; Satisfacción con la vida futura: $\left.\mathrm{F}_{(5 ; 309)=} 1.18, p=.316\right)$. Del mismo modo, tampoco se registraron diferencias en función del nivel de estudio de los participantes (Estereotipos sobre salud: $\mathrm{F}_{(5 ; 309)=} 5.75, p=.719$; Estereotipos sobre motivación: $\mathrm{F}_{(5 ; 309)=} 3.73, p=.876$; Estereotipos sobre personalidad: $\mathrm{F}_{(5 ; 309)=} 1.47, p=.197$; Temores hacia la vejez: $\mathrm{F}_{(5 ;}$ $309)=1.05, p=.386$; Satisfacción con la vida actual: $\mathrm{F}_{(5 ; 309)=} 1.78, p=.116$; Satisfacción con la vida futura: $\left.\mathrm{F}_{(5 ; 309)=} 1.89, p=.098\right)$.

\section{DISCUSIÓN}

El objetivo de este estudio fue analizar la relación entre los temores hacia el envejecimiento, los estereotipos de vejez, la satisfacción vital percibida y algunas variables socio-demográficas. Los resultados encontrados permiten concluir que la 
muestra bajo estudio cuenta con un nivel de estereotipos negativos bajo en las tres dimensiones evaluadas: salud, motivación y personalidad. Puede apreciarse que la tendencia en este estudio es similar a lo que se ha encontrado en estudios previos; vale decir que, de las tres dimensiones, la que hace referencia a las variables de personalidad es en la que se registran mayores estereotipos negativos (Lasagni Colombo et al., 2013).

Atendiendo a otras variables del estudio, tales como la convivencia con una persona mayor, los resultados muestran que existen diferencias estadísticamente significativas en los estereotipos negativos entre las personas que no conviven y aquellas otras que sí lo hacen; siendo marcadamente superiores en éste último grupo. Este podría deberse a las condiciones desfavorables de las personas mayores con las que conviven favorables, tales como cierto grado de dependencia o la presencia de alguna problemática física o mental. Esta situación podría intensificar los estereotipos negativos. En línea con tales conjeturas, diversos autores (Allan \& Johnson, 2009; Rello, Bravo \& Plata, 2018) han destacado que si las personas mayores conservan una funcionalidad óptima, los estereotipos negativos son menores.

En cuanto a las diferencias debidas al sexo, en este estudio no se han encontrado diferencias significativas entre varones y mujeres, coincidiendo de este modo con las conclusiones de otras investigaciones (Lasagni Colombo et al., 2013; León \& Montero, 2015). Lo que sí se ha evidenciado es que existe una relación estadísticamente significativa entre la edad y los estereotipos; aunque solo los que se relacionan con la dimensión de personalidad. En línea con las evidencias publicadas (Arnold-Cathalifaud et al., 2007; Lasagni Colombo et al., 2013) los hallazgos que se desprender del presente estudio indican que las personas de mayor edad muestran menos estereotipos negativos que las más jóvenes. Paralelamente, se ha encontrado una relación estadísticamente significativa e inversa entre la edad y los temores hacia el envejecimiento, de forma tal que las personas más jóvenes muestren mayores temores hacia el proceso de envejecimiento, quizás interiorizando los estereotipos existentes en la sociedad. Finalmente, analizando la relación entre los estereotipos y el nivel de satisfacción vital de los participantes, se ha encontrado que las personas con mayores estereotipos negativos hacia el envejecimiento muestran una menor satisfacción vital proyectada a cinco años. Posiblemente, porque perciban que, como consecuencia del proceso de envejecimiento, su calidad de vida actual pueda verse mermada. Este planteamiento está en la línea de lo apuntado por Amico (2009), al afirmar que los estereotipos negativos derivan en muchas ocasiones en temores hacia el propio proceso de envejecimiento, y dichos temores generan negación y hostilidad, derivando en insatisfacción. Esta afirmación coincide también en nuestro estudio, ya que se ha encontrado relación estadísticamente significativa y positiva entre los temores hacia el envejecimiento y los estereotipos hacia la vez. 
Esta información es muy relevante, ya que los jóvenes, que serán los mayores de mañana, continúan creyendo que ciertas creencias sobre el proceso de envejecimiento son ciertas, y por ello continúan perdurando en el tiempo, ayudadas muchas veces por la falta de contacto con personas mayores con una óptima funcionalidad. Todo ello repercute posteriormente en los temores que puedan tener sobre su propio proceso de envejecimiento. Este hecho podría estar en la línea del planteamiento de Ng, Allore, Trentalange, Monin y Levy (2015) que informan del aumento de estereotipos negativos hacia el proceso de envejecimiento. Sin embargo, otros autores parecen indicar que dicha concepción negativa se está modificando con el tiempo (Molino del Peral, 2000). Quizás pueda ser que en los jóvenes no se vayan eliminando del todo los estereotipos y posteriores miedos, pero que en los más mayores, más influenciados por la nueva concepción del envejecimiento activo, ya esté dando sus frutos.

Es muy importante que se analicen periódicamente y en diferentes contextos y edades, la asimilación de estos estereotipos negativos, para que se pueda intervenir en casos en los que sea necesario, adelantándonos a posibles problemáticas futuras que pueden darse en una sociedad que envejece a nivel global.

También son necesarias actuaciones que traten de fomentar el contacto entre personas mayores con alto grado de capacidad funcional y jóvenes de diferentes edades, ya que se ha demostrado que los estereotipos son menos negativos cuando se tiene contacto frecuente en dicas condiciones (Rello, Bravo y Plata, 2018; Özdemir y Bilgili ,2016; Bernardini et al. 2008; Elliott y Rubio, 2018).

Por todo ello, aunque se requieren más estudios que sigan arrojando luz sobre los estereotipos con el fin de erradicarlos, parece claro que la formación en envejecimiento sigue siendo la herramienta más potente para poder combatirlos (Allan y Johnson, 2009). De esta forma, pueden acercarse a aquellas personas de mayor edad cuya actividad social, calidad de vida, autoestima, ... en definitiva con un óptimo funcionamiento biopsicosocial está preservado (Kleinspehn-Ammerlahn, Kotter-Grühn, y Smith, 2008). Esto es fundamental ya que como se ha comentado, la exposición a determinados estereotipos negativos sobre el envejecimiento, puede predecir los resultados en las funciones físicas y cognitivas de las personas mayores (Oscanoa, Romero-Ortuno y Cruz-Jentoft , 2016).

También las personas mayores han de seguir teniendo acceso a la educación, pues aunque en este estudio no se ha mostrado la relación con el nivel educativo, estudios previos lo han reseñado, evidenciando que las personas con mayor nivel de estudios, muestra menores estereotipos negativos hacia el envejecimiento (Lasagni Colombo et al., 2013). Por ello, seguir garantizándoles el acceso a la educación, con el fin de seguir desarrollando sus capacidades, ayudará a prevenir la dependencia y a lograr un envejecimiento más pleno (Meneses, Sánchez-Serrano, Martínez y Galán, 2019). 
Estudios como el de Pinazo-Hernandiz, Torregrosa-Ruiz, Jimenez-Marti y Blanco-Molina (2019) demuestran la importancia de la participación de las personas mayores en actividades y de la relación que existe entre esta y la satisfacción con la vida, por lo que es fundamental favorecer la participación de las personas mayores en la comunidad, ya que este generará satisfacción y además les ayudará a derruir aquellos estereotipos negativos que continúen vigentes en su esquema mental.

\section{CONCLUSIONES}

Como conclusión a este estudio destacan los resultados obtenidos que muestran que no hay diferencias en ninguna de las variables estudiadas en función del sexo, pero sí se observan mayores estereotipos negativos entre quienes conviven con adultos mayores. La edad se asocia negativamente tanto a los estereotipos y temores como a la satisfacción con la vida. Finalmente, los estereotipos negativos sobre el envejecimiento se vinculan con menores niveles de satisfacción vital proyectada a cinco años. Por todo ello, sería fundamental que los programas de envejecimiento activo a todas las edades, trabajasen tomando como referencia aspectos como los comentados en este estudio.

Por último, cabe señalar que este estudio, aunque aporta una visión integradora de diferentes componentes relacionados con el envejecimiento, está realizado en un contexto determinado, por lo cual la generalización de los resultados ha de tomarse con cautela. Futuros estudios podrían estudiar la importancia de la participación en diferentes actividades cooperativas entre personas mayores y jóvenes y ver qué ocurre con los estereotipos en esos casos. También, otorgarle a las personas mayores más oportunidades de ocio y tiempo libre, vinculadas a la educación, puede repercutir en sus estereotipos, de tal forma que se pueda investigar el efecto de las diferentes actividades de ocio en la actividad cerebral, cognitiva, física y social

\section{Agradecimientos / Acknowledgments:}

Los autores agradecen la colaboración a todas las personas que participaron en este estudio.

\section{Rol de los autores / Authors Roles:}

VLF: realizó el diseño experimental, captación de la muestra, redacción, interpretación de resultados y corrección

SS: realizó procesamiento de los datos, análisis de los mismos y correcciones

KGR: recolección de la muestra, realizó interpretación de los resultados y correcciones 


\section{Fuentes de financiamiento / Funding:}

Este estudio no contó con ninguna fuente de financiación

\section{Aspectos éticos / legales; Ethics / legals:}

Las autoras declaran no haber incurrido en aspectos antiéticos respetando los códigos de investigación con humanos reseñados en los códigos deontológicos profesionales de España.

Los participantes firmaron el consentimiento informado el cual brindaba datos de la naturaleza, medios y objetivos de la investigación .

Se ha respetado lo establecido por las normativas éticas que regulan el ejercicio profesional (Código de Ética del Colegio de Psicólogos de España) Se procuró la protección de la confidencialidad de la información personal e institucional, asegurando el anonimato de las personas e instituciones involucradas en la muestra.

\section{Conflicto de intereses / Competing interests:}

Los autores no incurren en conflictos de intereses.

\section{REFERENCIAS}

Alvarado, A., \& Salazar, A. (2014). Análisis del concepto de envejecimiento. Gerokomos, 25(2), 57-62. http://dx.doi.org/10.4321/S1134-928X2014000200002

Allan, L. J., \& Johnson, J. A. (2009). Undergraduate attitudes toward the elderly: the role of knowledge, contact and aging anxiety. Educational Gerontology, 35(1), 1-14. http://dx.doi.org/10.1080/03601270802299780

Amico, L.C. (2009). Envejecer en el siglo XXI. No siempre Querer es Poder. Hacia la deconstrucción de mitos y la superación de estereotipos en torno a los adultos mayores en sociedad. Margen, 55, 1-30. Recuperado el 4 de abril de 2019 de https://www. margen.org/suscri/margen55/amico.pdf

Aristizábal-Vallejo, N., Morales, A., Salas, B. C., \& Torres, A. M. (2009). Estereotipos negativos hacia los adultos mayores en estudiantes universitarios. Cuadernos Hispanoamericanos de Psicología, 9(1), 35-44.

Arnold-Cathalifaud, M., Thumala, D., Urquiza, A., \& Ojeda, A. (2007). La vejez desde la mirada de los jóvenes chilenos estudio exploratorio. Última década, 15(27), 75-91

Ato, M., López. J., \& Benavente, A. (2013). Un sistema de clasificación de los diseños de investigación en psicología. Anales de Psicología, 29, 1038-1059. http://dx.doi.org/ 10.6018/analesps.29.3.178511

Bernardini, D. A., Moraru, M., Hanna, M., Kalache, A., \& Macias, J. F. (2008). Attitudes towards the elderly among university students of health care related studies at the University of Salamanca, Spain. Journal of Continuing Education in the Health Professions, 28(2), 86-90. http://dx.doi.org/10.1002/chp.162 
Brotons Rodes, P., Lorente Martínez, R., \& Sitges Maciá, E. (2020). Efectos del Programa ACOMPAÑA-TÉ sobre los estereotipos hacia la vejez en universitarios. Revista de Psicología de la Salud, 8, (1), 1-43. https://doi.org/10.21134/pssa.v8i1.1504

Díaz, I. P. B., Martínez, H. L., Colín, C. A. M., González, D. A. O., Herrera, A. A. S., Carbajal, A. J. Z. et al. (2016). La percepción de los adolescentes hacia la vejez. Revista Huella de la Palabra, (9), 83-93.

Dionigi, R. A. (2015). Stereotypes of aging: their effects on the health of older adults. Journal of Geriatrics [Versión electrónica]. Recuperado el 14 de junio de 2017 de http://dx.doi.org/10.1155/2015/954027

Elliott, P. V., \& Rubio, L. (2018). Cambios en los estereotipos sobre la vejez de estudiantes tras su participación en un proyecto intergeneracional. International Journal of Developmental and Educational Psychology. Revista INFAD de Psicología, 1(2), 61-68. https://doi.org/10.17060/ijodaep.2017.n2.v1.1108

Fundación Humanismo y Ciencia. Temores hacia el envejecimiento). Recuperado el 10 de marzo de 2017 http://fun-humanismo-ciencia.es/felicidad/mayor/mayorl.htm

González Bernal, J., \& de la Fuente Anuncibay, R. (2014). Desarrollo humano en la vejez: un envejecimiento optimo desde los cuatro componentes del ser humano. International Journal of Developmental and Educational Psychology, 7 (1), 121-129. https:// doi.org/10.17060/ijodaep.2014.n1.v7.783

Kleinspehn-Ammerlahn, A., Kotter-Grühn, D., \& Smith, J. (2008). Self-perceptions of aging: do subjective age and satisfaction with aging change during old age. The Journals of Gerontology, 63(6), 377-385. http://dx.doi.org/10.1093/geronb/63.6.p377

Jansen, F., \& Vogel, N. (2007). Percepciones en torno al envejecimiento en jóvenes universitarios: apuntes en torno a la profecía autocumplida. VI Congreso Chileno de Antropología, Valdivia. Recuperado el 13 de junio de 2017 de https://www.aacademica. org/vi.congreso.chileno.de.antropologia/25.pdf

Lasagni Colombo, V. X., Rodríguez Bessolo, M. S., Palermo Guiñazu, N., Gutiérrez, T., Luynés, M., Crespo Tarifa, E., ... \& Díaz Veiga, P. (2013). Estereotipos hacia la Vejez en Adultos Mayores y en Estudiantes Universitarios en diez países de América Latina. Recuperado el 1 de abril de 2017 https:/fiapam.org/wp-content/uploads/2013/10/ ESTEREOTIPOS-LATINOAMERICA-FINAL.pdf

León, O. G., \& Montero, I. (2015). Métodos de investigación en psicología y educación. Las tradiciones cuantitativas y cualitativas. Madrid, España: McGraw-Hill.

López Gómez, M., \& Marín Baena, R. (2016). Revisión teórica y empírica desde la psicología sobre representaciones sociales del envejecimiento y la vejez en Latinoamérica y España (2009-2013). Revista Científica General José María Córdova, 14 (17), 155-202.

Meneses, E. L., Sánchez-Serrano, J. L. S., Martínez, A. J., \& Galán, J. G. (2019). Percepción de los estudiantes de posgrado en gerontología sobre el envejecimiento. Revista de Humanidades, (36), 155-180. 
Ng, R., Allore, H. G., Trentalange, M., Monin, J. K., \& Levy, B. R. (2015). Increasing negativity of age stereotypes across 200 years: evidence from a database of 400 million words. PLoSOne, 10(2), 10-15. http://dx.doi.org/10.1371/journal.pone.0117086

Monchietti, A., Lombardo, E., \& Sánchez, M. (2015). Representación social de la vejez en niños y púberes. Limite: Revista Interdisciplinaria de Filosofía y Psicología, 2(16), $71-81$.

Oscanoa, T., Romero-Ortuno, R., \& Cruz-Jentoft, A. (2016). Estereotipos negativos asociados a la fragilidad en personas mayores. Revista Española de Geriatría y Gerontología, 51(4), 225-228. http://dx.doi.org/10.1016/j.regg.2016.01.011

Özdemir, Ö., y Bilgili, N. (2016). Attitudes of Turkish nursing students related to ageism. Journal of Nursing Research, 24(3), 211-216. http://dx.doi.org/10.1097/ jnr.0000000000000131

Pearman, A., Hertzog, C., \& Gerstorf, D. (2014). Little evidence for links between memory complaints and memory performance in very old age: Longitudinal analyses from the berlin aging study. Psychology and Aging, 29(4), 828-842. http://dx.doi. org/10.1037/a0037141

Perea, I. (2018). Acción colectiva de las mujeres y procesos emancipadores en América Latina y el Caribe: una aproximación desde los casos de Cuba, Bolivia y Ecuador. Foro Int [en línea]. [citado 1 Abr 2019]; 57(4):915-950. Disponible en: http://www. scielo.org.mx/pdf/fi/v57n4/0185-013X-fi-57-04-00915.pdf

Pinazo-Hernandiz, S., Torregrosa-Ruiz, M., Jimenez-Marti, M., \& Blanco-Molina, M. (2019). Participación social y satisfacción vital: tipos de participación y diferencias entre mujeres y hombres mayores. Revista de psicología de la salud, 7(1), 202-234.

Rello, C. F., Bravo, M. D. L., \& Plata, R. M. M. (2018). Estereotipos sobre la edad y el envejecimiento en estudiantes y profesionales de Ciencias de la Salud. Prisma Social: Revista de Investigación Social, (21), 108-122.

Rojas, M. (2008). Bienestar Subjetivo en Costa Rica. Decimocuarto informe estado de la nación en desarrollo humano sostenible. Recuperado el 23 de abril de 2017 de https:// estadonacion.or.cr/files/biblioteca_virtual/014/Bienestar-subjetivo-Rojas-2008.pdf 